\title{
Overexpression of MyoD Attenuates Denervated Rat Skeletal Muscle Atrophy and Dysfunction*
}

\author{
Weimin Xiong ${ }^{1}$, Jiahui Huang ${ }^{2}$, Lei Xie ${ }^{2}$, Yuan Qiao ${ }^{2}$, Xinmin Lü ${ }^{2}$, Jiangchen Peng ${ }^{2}$, Jinjia Hu ${ }^{2 \#}$ \\ ${ }^{1}$ Department of Gastroenterology, Shanghai 445 Hospital, Shanghai, China; ${ }^{2}$ Medical College of Shanghai Jiao Tong University, \\ Shanghai, China. \\ Email: \#jhu2609@126.com
}

Received August $28^{\text {th }}, 2012$; revised September $28^{\text {th }}, 2012$; accepted October $7^{\text {th }}, 2012$

\begin{abstract}
Nerve injury commonly contributes to irreversible functional impairment, reconstruction of the function of muscle is big challenge. In denervated skeletal muscle, therapid expression of MyoD mRNA and protein also occurs during early postdenervation, which suggested that the function of denervation-induced MyoD may be to prevent denervation-induced skeletal muscle atrophy. However, the detail mechanism is not clear. Therefore, in this study, we established a stable-transfected MyoD L6 cell line. After the operation for cutting the rats' tibial nerve, the MyoD-L6 cells were injected in the gastrocnemius, the function of the gastronemius was monitored. It was found that injected the MyoD-L6 cells could attenuate the muscle atrophy and dysfunction. Therefore, overexpression of MyoD could serve as a new therapy strategy to cure denervation-induced dysfunction of skeletal muscle.
\end{abstract}

Keywords: MyoD; Denervation; Rat Skeletal Muscle; Atrophy; Dysfunction

\section{Introduction}

Nerve injury contributes to irreversible functional impairment, for example, destroying the nerve controlling skeletal muscle will result in skeletal muscle atrophy and dysfunction, which will seriously affect patients' lives $[1,2]$. To find a feasible solution, the research work in the world main focus on three fields: firstly, shortening the distance the nerve growth; Secondly, promoting nerve growth; On the other hand, attenuating the denervated skeletal muscle atrophy [1,3-6]. In clinical work, we commonly observed that nerve injury lead to the dysfunction of skeletal muscle because the muscle has already became to fibering before the nerve regeneration [7]. Therefore, if an effective solution to attenuate skeletal muscle atrophy until the injury nerve regeneration, it will abolish the dysfunction of skeletal muscle resulted by denervation.

MyoD is a member of the basic-helix-loop-helix family of proteins and acts as a transcriptional factor in several skeletal muscle-specific genes [8-10]. In normal embryonic development MyoD is upregulated at the time when the hypaxial musculature begins to form, but its role in the denervated skeletal muscle remains to be elucidated. It was reported that $\mathrm{MyoD}^{-/-}$myogenic cells are committed to myoblast and proliferation. It was ob-

\footnotetext{
We declare we have no any interest conflict.

${ }^{\#}$ Corresponding author.
}

served that MyoD in vivo is expressed in adult skeletal muscle in response to diverse stimuli, such as overload, injury, andexercise. Especially in the early stage of nerve injury, the increasing expression of MyoD was observed, which suggested that MyoD plays a role in the plasticity of skeletal muscles. In denervated skeletal muscle, therapid expression of MyoD mRNA and protein also occurs during early postdenervation. Several studies suggested that the function of denervation-induced MyoD may be to prevent denervation-induced muscle atrophy [7,11-14]. However, rare information that MyoD prevents skeletal muscle atrophy could be obtained, and the molecular mechanism is not clear.

Therefore, our goal in this study is to investigate the effect of overexpression of MyoD skeletal muscle on attenuating denervation-induced muscle atrophy, and wish this will provide a new therapy to cure denervation-induced dysfunction of skeletal muscle.

\section{Materials and Methods}

\subsection{Rat Muscle Cell Line and Cell Culture}

Rat skeletal muscle L6 cells were obtained from ATCC and cultured in MEM supplemented with 10\% FBS, 10 $\mathrm{mM}$ HEPES buffer, $2 \mathrm{mM}$ L-Glutamine, $50 \mathrm{U} / \mathrm{ml}$ penicillin and $50 \mathrm{mg} / \mathrm{ml}$ streptomycin at $37^{\circ} \mathrm{C}$ in a humidified atmosphere with $5 \% \mathrm{CO}_{2}$. Upon reaching confluence, 
differentiation was induced by media containing $2 \%(\mathrm{v} / \mathrm{v})$ fetal bovine serum for 7 days.

\subsection{Total RNA Extraction and Real-Time Quantitative RT-PCR Analysis}

Total RNA from cells or rat skeletal muscle was prepared using TRIZOL, according to the manufacture's instructtions. Total RNA $1 \mu \mathrm{g}$ was reverse transcribed into cDNA in a $20 \mu 1$ reaction. Briefly, the RNA solution was incubated at $70^{\circ} \mathrm{C}$ for $5 \mathrm{~min}$ and then chilled on ice, following all samples were incubated at $37^{\circ} \mathrm{C}$ for $1 \mathrm{~h}$ in 20 ul of $10 \mathrm{mM}$ TRIS (pH 8.4), $50 \mathrm{mM} \mathrm{KCl}, 2 \mathrm{mM} \mathrm{MgCl}, 1$ $\mathrm{mM}$ DTT, 5 units of ribonuclease inhibitor, $0.5 \mathrm{mM}$ dNTP, 100 pmol of oligo-d(T) 18 and 200 units of MMLV reverse transcriptase (Promega Company, USA). The reaction was terminated by heating for $5 \mathrm{~min}$ at $95^{\circ} \mathrm{C}$. Reverse transcribed products were used for quantitative real-time PCR amplification.

The primers used for SYBR Green real-time RT-PCR are as follows: MyoD, sense,

5'-GACGAAGTCTGGTTGTTGTTGC-3'; antisense, 5'-GCTAGGGACTGTGAGGAAAGGA-3'; $\beta$-actin, sense, 5'-GAAATCGTGCGTGACATTA-3'; antisense,

5'-TAGGAGCCAGGGCAGTAA-3'. These primers were purchased from Sangon company (Sangon, Shanghai, China). The reactions were set up with $10 \mu \mathrm{l}$ SYBR Green PCR Master Mix (Takara, Shuzo, Kyoto, Japan), $1.0 \mu 110 \mu \mathrm{mol}$ primer mixture, and $2 \mu \mathrm{lcDNA}$ template. Real time PCR conditions were as follows: $95^{\circ} \mathrm{C}$ for 15 seconds followed by 30 cycles of $54^{\circ} \mathrm{C}(\mathrm{MyoD})$ or $57^{\circ} \mathrm{C}$ $\beta$-actin) for 30 seconds and $72^{\circ} \mathrm{C}$ for 30 seconds. Water was used as a negative control. Relative gene expression was determined by the fluorescence intensity ratio of the target genes to $\beta$-actin.

\subsection{Western Blot Analyses}

All the muscle samples collected were lysed in lysis buffer $(0.5 \%$ Nonidet P(NP)-40, $10 \mathrm{mM}$ Tris, $\mathrm{pH} 7.4$, $150 \mathrm{mM} \mathrm{NaCl}, 1 \mathrm{mM}$ ethylene diamine tetra-acetic acid [EDTA], $1 \mathrm{mM} \mathrm{Na}_{3} \mathrm{VO}_{4}$ containing protease inhibitors (1 $\mathrm{mM}$ phenylmethylsulfonyl fluoride [PMSF]). Proteins were loaded on a $10 \%$ or $12 \%$ SDS-PAGE, followed they were transferred to polyvinylidene fluoride (PVDF) membranes and blocked in 5\% non-fat milk in $10 \mathrm{mM}$ Tris, pH 7.5, $100 \mathrm{mM} \mathrm{NaCl}, 0.1 \%$ (w/v) Tween-20 for 2 hrs. Anti-human MyoD and $\beta$-actin rabbit polyclonal antibody were diluted at $1: 1000$ and incubated at $4{ }^{\circ} \mathrm{C}$ overnight, followed by $1 \mathrm{hr}$ incubation with the appropriate secondary antibody. The blots were further developed using the chemiluminiscence reagent.

\subsection{Plasmid Construction and Transfection}

The MyoD gene was amplified and the PCR product was collected and purified, then was inserted into the EcoRIBamHI site of pLVX to generate the pMyoD-LVX plasmid. The primers were designed as follows: sense, 5'CCGGAATTCATGGAGCTACTATCGCCGCCAC-3', which including EcoR I restriction enzyme cutting site; antisense,

5'-CGCGGATCCTCAGAGCACCTGGTAAATCGGATTG-3', which including BamHI restriction enzyme cutting site. The integrity of the cDNA was confirmed by sequencing. $8 \mu \mathrm{g}$ pMyoD- LVX and $8 \mu \mathrm{g}$ empty vector were transfected into L6 cells using Lipofectamine 2000 (Invitrogen) according to the instructions provided by the manufacturer.

\subsection{Production of Lentiviral Particles}

In order to prepare lentiviral particles expressing the MyoD gene, HEK-293T cells were transfected with pMyoD-LVX plus lentiviral packaging vectors. Briefly, the cells were seeded in a 6-well plate at a concentration of $1.0 \times 10^{6}$ cells per well. After $24 \mathrm{~h}$, the culture medium was aspirated and replaced with Opti-MEM. Subsequently, $2 \mu \mathrm{g}$ pMyoD- LVX and the Mission Lentiviral Packaging Mix (Sigma) were transfected into cells by Lipofectamine 2000 (Invitrogen) according to the manufacturer's instructions. On the following day, the culture was replaced with complete medium (DMEM with 10\% FBS). After another $48 \mathrm{~h}$, the culture supernatants containing the lentiviral particles were harvested for use.

\subsection{Establishment of MyoD-Overexpressing L6 Cell Lines}

The L6 cells were transduced with the Lenti-MyoD lentiviral particles. On the third day after transduction, puromycin (Sigma-Aldrich) was added into the culture medium to a final concentration of $2 \mu \mathrm{g} / \mathrm{ml}$. During the selection period, the drug was kept at the same concentration at each replacement of culture medium. Approximately 2 weeks was required for the live cells to be eliminated in the mock transduction group. After that, the selected cultures were expanded and cryopreserved. The MyoD mRNA was determined by real time PCR.

\subsection{Animal Treatment}

30 rats about $200 \mathrm{~g} / \mathrm{each}$ were used to determine the effect of overexpression of MyoD on denervated rat skeletal muscle atrophy and dysfunction. To construct denervation model, 30 rats were cut tibial nerve after anesthesia. Four weeks latter, these rats were devided into 5 groups and four groups were injected in extensor digitorum longus at several differential places with the cells transfected with Lentivurus-MyoD-L6, Lentivurus-MyoD, Lentivurus-L6, and Lentivurus, respectively. Four weeks latter, the denervation group, Lentivurus-MyoD-L6 and 
Lentivurus-MyoD groups were performed dialyneury, the wet weight of gastrocnemius and contraction forces were determined.

\subsection{Statistical Analyses}

The results of real time PCR, wet weight of gastrocnemius and contraction forces were analyzed with Student's test. A two-sided test with $P$ values of less than 0.05 was considered statistically significant.

\section{Results}

\subsection{MyoD Overexpressed in Stable-Transfected MyoD L6 Cells}

To investigate the role MyoD on the function repair of skeletal muscle, the stable-transfected MyoD L6 cells were established by Lentivirus transduction. The real time PCR was performed to determine the relative expression of MyoD mRNA after transfection. As showed in Figure 1, the L6 cells stable-transfected MyoD showed significant increase MyoD mRNA, compared with the control cells.

\subsection{Transfection of MyoD Induced MyoD Expression in Denervated Rats}

The rats were performed the operation to cut the tibial nerve after anesthesia, 4 weeks later, stable-transfect MyoD L6 cells (MyoD-L6), Lenti-MyoD lentiviral particles (MyoD), L6 cells (L6) and leti-empty vector particles (vector) were injected in the denervated skeletal muscles, respectively. The MyoD mRNA and protein were examined after another 4 weeks or 6 weeks, it was observed that mRNA potently increased in MyoD-L6 group (Figures 2(a) and (b)), however, similar expression pattern in protein level was not observed after injecttion for 4 weeks (Figure 3(a)). Consistent increase MyoD protein in MyoD-L6 group was observed after injection for 6 weeks (Figure 3(b)).

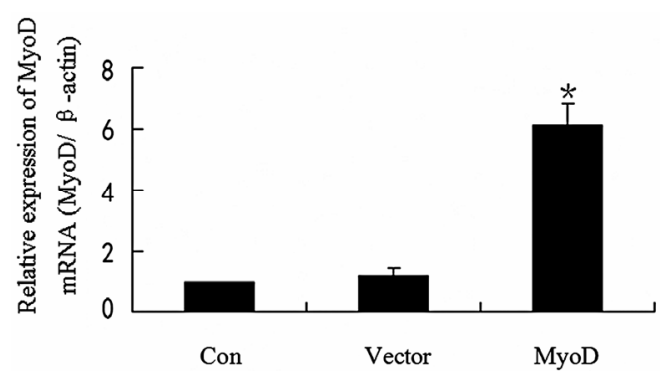

Figure 1. MyoD mRNA increase in stable-transfect MyoD L6 cells. Real time PCR was performed to examine MyoD mRNA expression in L6 cells, stable-transfect empty vector L6 cells and stable-transfect MyoD L6 cells, each experiment repeat three times. Beta action serves as a loading control.

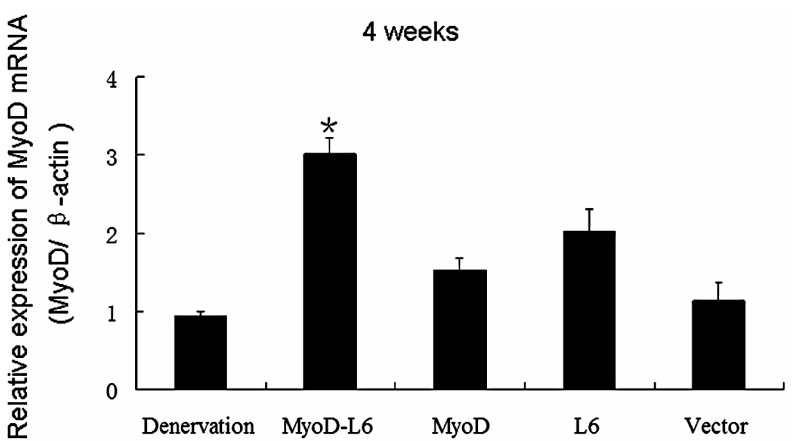

(a)

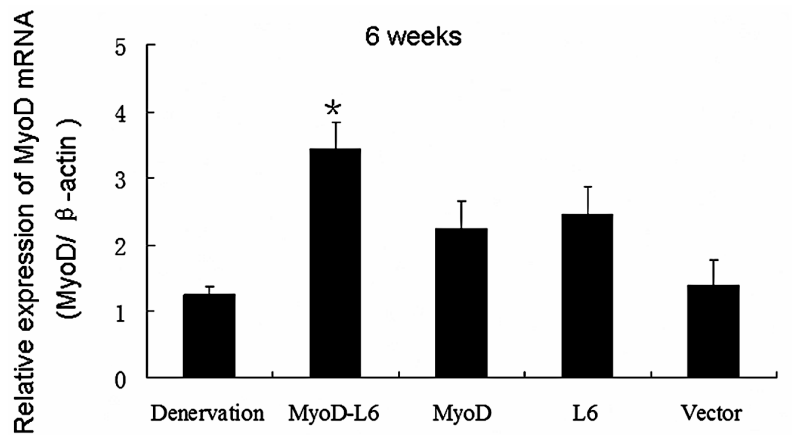

(b)

Figure 2. The MyoD mRNA expression in denervated rats gastrocnemius muscle. The denervated rats were injected with stable-transfect MyoD L6 cells (MyoD-L6), LentiMyoD lentiviral particles (MyoD), L6 cells (L6) and letiempty vector particles (vector), respectively. MyoD mRNA were determined in 4 weeks or 6 weeks after injection by real time PCR, each experiment was repeat three times.

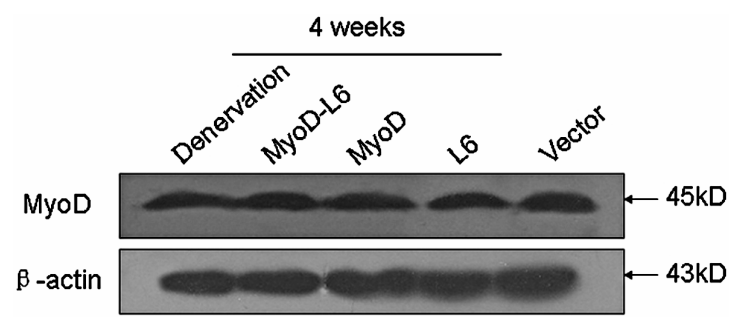

(a)

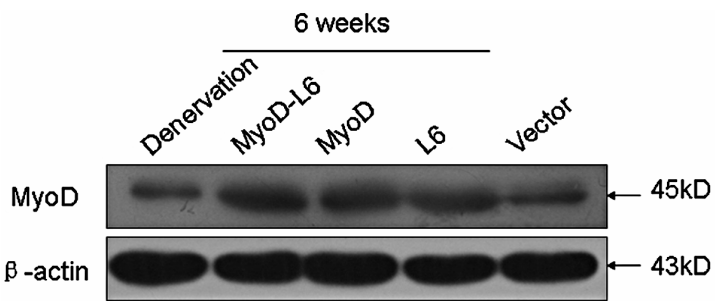

(b)

Figure 3. The MyoD protein expression in denervated rats gastrocnemius muscle. The denervated rats were injected with stable-transfect MyoD L6 cells (MyoD-L6), LentiMyoD lentiviral particles (MyoD), L6 cells (L6) and letiempty vector particles (vector), respectively. MyoD mRNA were determined in 4 weeks or 6 weeks after injection by Western blot, each experiment was repeat three times. 


\subsection{Overexpression MyoD Attenuated Denervated Skeletal Muscle Atrophy}

From the Figure 4, we can see that the wet weight of denervated skeletal muscle in MyoD-L6 group was more weight than other goups after performing denervation operation for 4 weeks or 6 weeks. The rats were injected with stable-transfect MyoD L6 cells (MyoD-L6), LentiMyoD lentiviral particles (MyoD), L6 cells (L6) and letiempty vector particles (vector) after denervation, respecttively, after 4 weeks of injection, the weight of skeletal muscles were examined in different time. It was found that the wet weight of denervated skeletal muscle in MyoD-L6 group was more weight than other groups (Figure 4), which indicated that overexpressed MyoD retard denervated skeletal muscle atrophy. Meanwhile, after 4 weeks of nerve repair, we found that overexpression of MyoD significantly regarded the atrophy after nerve repair for 12 weeks (Figure 7).

\subsection{Overexpression MyoD Attenuated Denervated Skeletal Muscle Dysfunction after Reconstruction of Nerve}

To investigate the role of MyoD on function repair after reconstruction of nerve, the contraction forces were determined. As we observed that in Figures 5, 6, 8 and 9,

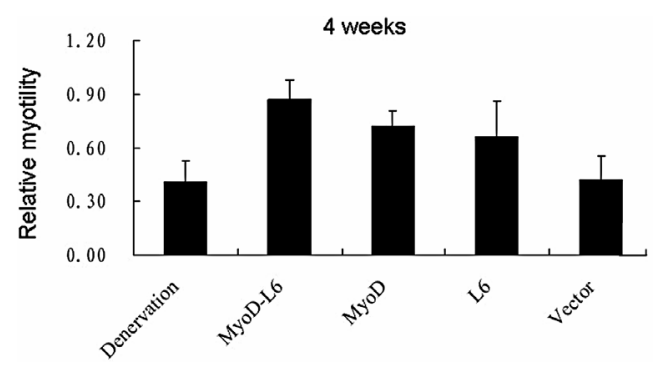

(a)

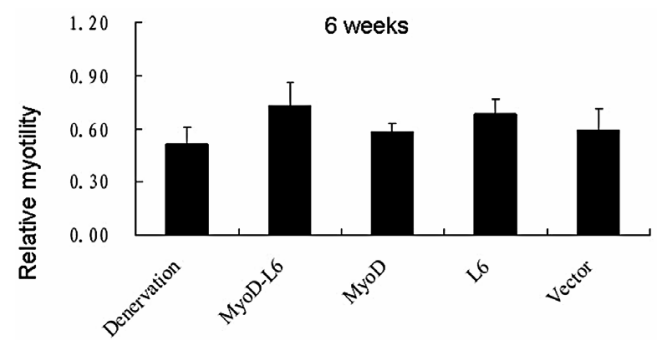

(b)

Figure 4. The single myotility of denervated rats gastrocnemius muscle. The denervated rats were injected with stable-transfect MyoD L6 cells (MyoD-L6), Lenti-MyoD lentiviral particles (MyoD), L6 cells (L6) and leti-empty vector particles (vector), respectively. The single motility was determined in 4 weeks or 6 weeks after injection, the relative single motility was shown as the single motility of the gastrocnemius muscle underwent denervation compared with that of gastrocnemius muscle not underwent denervation.

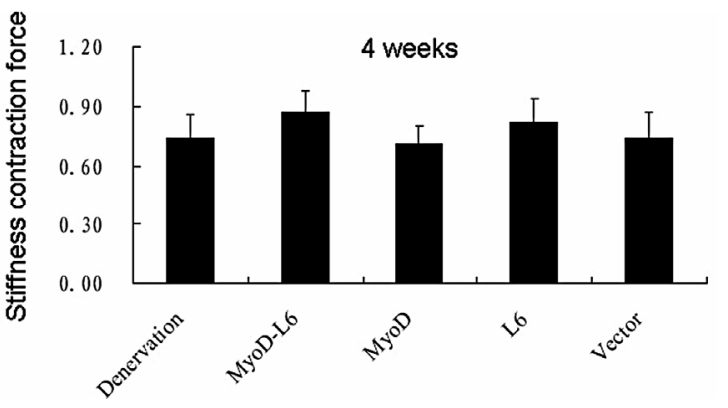

(a)

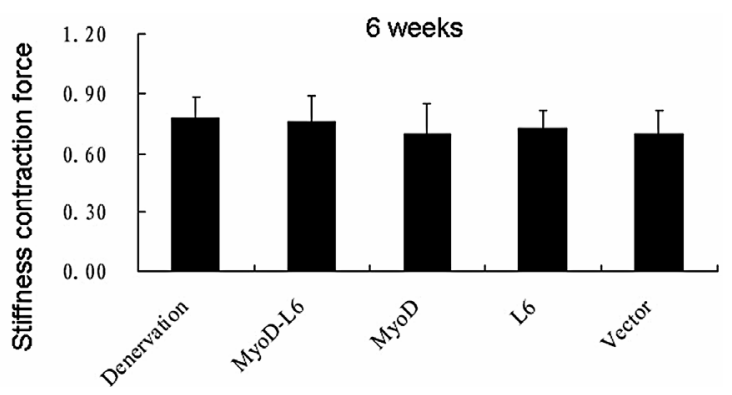

(b)

Figure 5. The max stiffness contraction force of denervated rats gastrocnemius muscle. The denervated rats were injected with stable-transfect MyoD L6 cells (MyoD-L6), Lenti-MyoD lentiviral particles (MyoD), L6 cells (L6) and leti-empty vector particles (vector), respectively. The max stiffness contraction force was determined in 4 weeks or 6 weeks after injection, the relative max stiffness contraction force was shown as the max stiffness contraction force of the gastrocnemius muscle underwent denervation compared with that of gastrocnemius muscle not underwent denervation.

the single contraction forces and siffness contraction force in MyoD-L6 group were stronger than other groups after reconstruction of nerve, which suggested that overexpression $\mathrm{MyoD}$ attenuate denervated skeletal muscle dysfunction after reconstruction of nerve.

\section{Discussion}

Effective nerve repair could recover denervated skeletal muscle function in some extent, commonly, skeletal muscle have already been fibrosis and lose its function before the nerve repair. Thus, retarding denervated skeletal muscle atrophy until the nerve repair is a feasible solution to cure the muscle hurt results from denervation. However, attenuating muscle atrophy is a challenge and detail mechanism remains to be clarified.

It was reported that skeletal muscle satellite cells are tissue-specific stem cells that are responsible for skeletal muscle growth and proliferation. In response to some injury, satellite cells re-enter the cell cycle, activate expression of muscle regulatory factors, such as $\mathrm{MyoD}$ and Myf-5, and execute the myogenic program, finally, restoring muscle structure and function [15-18]. Several 


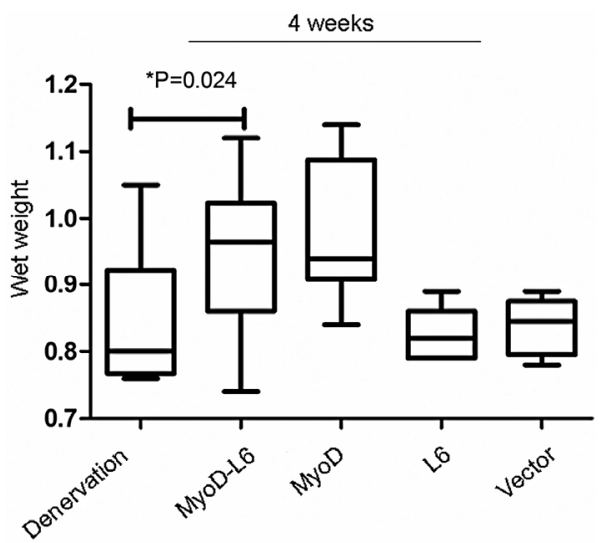

(a)

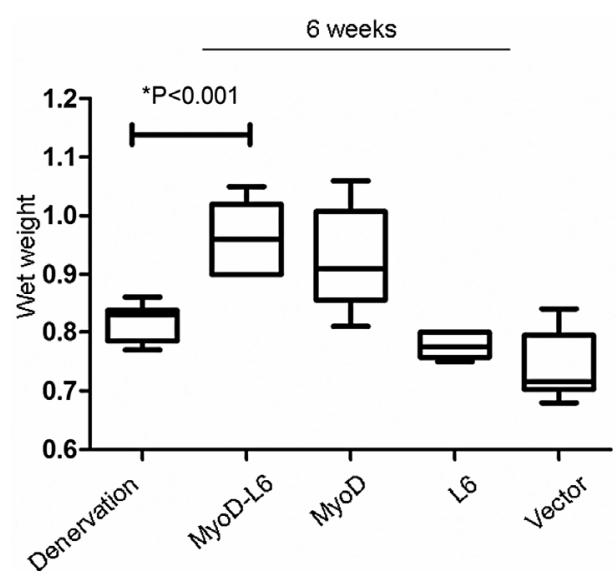

(b)

Figure 6. The wet weight of denervated rats gastrocnemius muscle. The denervated rats were injected with stabletransfect MyoD L6 cells (MyoD-L6), Lenti-MyoD lentiviral particles (MyoD), L6 cells (L6) and leti-empty vector particles (vector), respectively. The wet weight was determined in 4 weeks or 6 weeks after injection, the relative wet weight was shown as the wet weight of the gastrocnemius muscle underwent denervation compared with that of gastrocnemius muscle not underwent denervation.

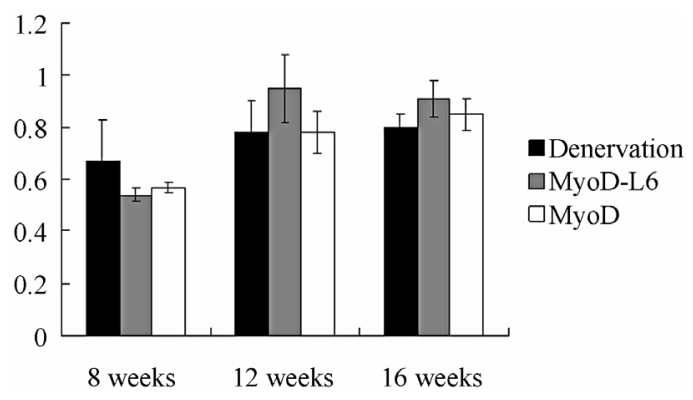

Figure 7. Overexpression of MyoD regards denervated rats gastrocnemius muscle's atrophy after nerve repair. The denervated rats were injected with stable-transfect MyoD L6 cells (MyoD-L6), Lenti-MyoD lentiviral particles (MyoD), respectively. After transfection 4 weeks latter, the nerve was repaired, the wet weight of gastrocnemius muscle was examined in 8 weeks, 12 weeks and 16 weeks after nerve repairing, respectively.

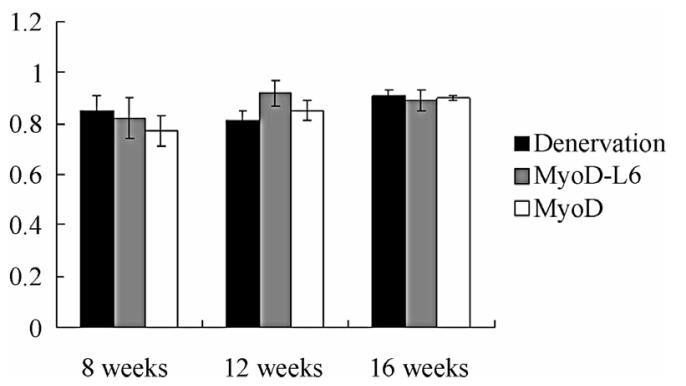

Figure 8. Overexpression of MyoD regards denervated rats gastrocnemius muscle's loseness of single myotility after nerve repair. The denervated rats were injected with stable-transfect MyoD L6 cells (MyoD-L6), Lenti-MyoD lentiviral particles (MyoD), respectively. After transfection 4 weeks latter, the nerve was repaired, the single myotility of gastrocnemius muscle was examined in 8 weeks, 12 weeks and 16 weeks after nerve repairing, respectively.

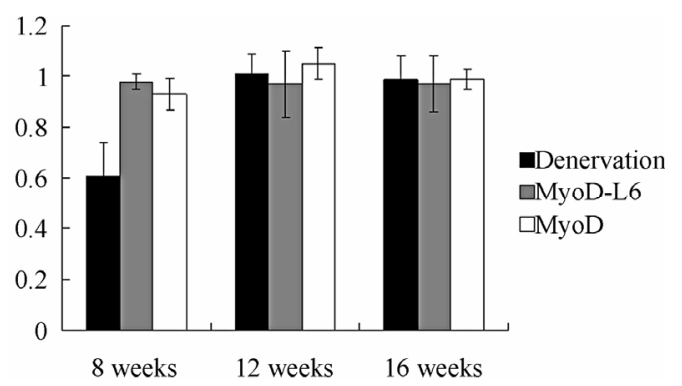

Figure 9. Overexpression of MyoD regards denervated rats gastrocnemius muscle's loseness of max stiffness contraction force after nerve repair. The denervated rats were injected with stable-transfect MyoD L6 cells (MyoD-L6), LentiMyoD lentiviral particles (MyoD), respectively. After transfection 4 weeks latter, the nerve was repaired, the max stiffness contraction force of gastrocnemius muscle was examined in 8 weeks, 12 weeks and 16 weeks after nerve repairing, respectively.

study indicated that skeletal muscle satellite cell increased and MyoD overexpressed at the early stage of nerve hurt [15,18-20], these evidence suggested that satellite cells could transform to adult skeletal muscle to protect the muscle from dysfunction.

In current study, we established a stable-transfect MyoD L6 cells, and investigated the role of MyoD on retarding skeletal muscle atrophy. Compared with the rats with denervation, the groups combinational denervation with MyoD L6 cells transfection, or the group combinational denervation with MyoD transfection, the expressions of MyoD mRNA and protein significantly increased after injecting these cells for 4 or 6 weeks. Most importantly, the wet weight of denervated skeletal muscle in these groups overexpressed MyoD was potently more weight than the single denevation group, which indicated that overexpressed MyoD retards denervated skeletal muscle atrophy. On the other hand, we investigated the contraction forces of these rats after nerve repair, that is to say 
determine the denervated skeletal muscle function after reconstruct the never system. We found that the rats overexpressed MyoD have more strong contraction forces. Regeneration of adult skeletal muscle is an asynchronous process requiring the activation, proliferation and fusion of satellite cells, to form new muscle fibres. Previous study indicated that activated satellite cells, which express either Myf5 or MyoD, do not accumulate selectively on fast or slow muscle fibres. But the activated satellite cells with overexpressed MyoD may increase the cell number of adult skeletal muscle though it did not affect the muscle fibres form. It was also reported that Primary $\mathrm{MyoD}^{-/-}$myogenic cells exhibited a stellate morphology distinct from the compact morphology of wild-type myoblasts, and expressed c-met, a receptor tyrosine kinase expressed in satellite cells. However, $\mathrm{MyoD}^{-/-}$myogenic cells did not express desmin, an intermediate filament protein typically expressed in cultured myoblasts in vitro and myogenic precursor cells in vivo, which indicated that deficit of MyoD could form adult muscle. Base on our study and other research, we could safely to say that overexpressed MyoD facilitate denavated skeletal muscle function repair.

In summary, it was found that increase MyoD molecule could retard denervated rat skeletal muscle atrophy and dysfunction, which may provide a new therapy strategy for the patients who suffers skeletal muscle dysfunction resulted from denervation.

\section{Acknowledgements and Interest Conflict}

The work was supported by grant of Science and Technology Innovation Subject from Nanjing Milotary Region (No. 08MA035).

\section{REFERENCES}

[1] P. J. Plant, J. R. Bain, J. E. Correa, M. Woo and J. Batt, "Absence of Caspase-3 Protects Against Denervation-Induced Skeletal Muscle Atrophy," Journal of Applied Physiology, Vol. 107, No. 1, 2009, pp. 224-234. doi:10.1152/japplphysiol.90932.2008

[2] P. M. Siu and S. E. Always, "Response and Adaptation of Skeletal Muscle to Denervation Stress: The Role of Apoptosis in Muscle Loss," Frontiers in Bioscience, Vol. 14, 2009, pp. 432-452. doi: $10.2741 / 3253$

[3] B. C. Beehler, P. G. Sleph, L. Benmassaoud and G. J. Grover, "Reduction of Skeletal Muscle Atrophy by a Proteasome Inhibitor in a Rat Model of Denervation," Experimental Biology and Medicine (Maywood), Vol. 231, No. 3, 2006, pp. 335-341.

[4] F. L. Muller, W. Song, et al., "Denervation-Induced Skeletal Muscle Atrophy is Associated with Increased Mitochondrial ROS Production," American Journal of Physiology: Regulatory, Integrative and Comparative Physiology, Vol. 293, No. 3, 2007, pp. R1159-R1168. doi:10.1152/ajpregu.00767.2006
[5] S. L. Rowan, K. Rygiel, F. M. Purves-Smith, N. M. Solbak, D. M. Turnbull and R. T. Hepple, "Denervation Causes Fiber Atrophy and Myosin Heavy Chain Co-Expression in Senescent Skeletal Muscle," PLoS One, Vol. 7, No. 1, 2012, p. e29082. doi:10.1371/journal.pone.0029082

[6] T. Shavlakadze, J. D. White, M. Davies, J. F. Hoh and M. D. Grounds, "Insulin-Like Growth Factor I Slows the Rate of Denervation Induced Skeletal Muscle Atrophy," Neuromuscular Disorders, Vol. 15, No. 2, 2005, pp. 139. 146. doi:10.1016/j.nmd.2004.10.013

[7] M. Ishido, K. Kami and M. Masuhara, "In Vivo Expression Patterns of MyoD, P21, and Rb Proteins In myonuclei and Satellite Cells of Denervated Rat Skeletal Muscle," American Journal of Physiology: Cell Physiology, Vol. 287, No. 2, 2004, pp. C484-C493. doi:10.1152/ajpcell.00080.2004

[8] A. Buonanno, L. Apone, M. I. Morasso, R. Beers, H. R. Brenner and R. Eftimie, "The MyoD Family of Myogenic Factors is Regulated by Electrical Activity: Isolation and Characterization of a Mouse Myf-5 CDNA," Nucleic Acids Research, Vol. 20, No. 3, 1992, pp. 539-544. doi:10.1093/nar/20.3.539

[9] J. P. Hyatt, R. R. Roy, K. M. Baldwin and V. R. Edgerton, "Nerve Activity-Independent Regulation of Skeletal Muscle Atrophy: Role of MyoD and Myogenin in Satellite Cells and Myonuclei," American Journal of Physiology: Cell Physiology, Vol. 285, No. 5, 2003, pp. C1161-C1173.

[10] K. Koishi, M. Zhang, I. S. McLennan and A. J. Harris, "MyoD Protein Accumulates in Satellite Cells and Is Neurally Regulated in Regenerating Myotubes and Skeletal Muscle Fibers," Developmental Dynamics, Vol. 202, No. 3, 1995, pp. 244-254. doi:10.1002/aja.1002020304

[11] S. B. Charge, A. S. Brack, S. A. Bayol and S .M. Hughes, "MyoD- and Nerve-Dependent Maintenance of MyoD Expression in Mature Muscle Fibres Acts through the DRR/PRR Element," BMC Developmental Biology, Vol. 8, 2008, p. 5. doi:10.1186/1471-213X-8-5

[12] M. Ekmark, Z. A. Rana, G. Stewart, D. G. Hardie and K. Gundersen, "De-Phosphorylation of MyoD is Linking Nerve-Evoked Activity to Fast Myosin Heavy Chain Expression in Rodent Adult Skeletal Muscle," The Journal of Physiology, Vol. 584, Pt. 2, 2007, pp. 637-650. doi:10.1113/jphysiol.2007.141457

[13] K. Sakuma, K. Watanabe, M. Sano, I. Uramoto, K. Sakamoto and T. Totsuka, "The Adaptive Response of MyoD Family Proteins in Overloaded, Regenerating and Denervated Rat Muscles," Biochimica et Biophysica Acta, Vol. 1428, No. 2-3, 1999, pp. 284-292. doi:10.1016/S0304-4165(99)00086-0

[14] Z. Wu, H. Jin and Y. Gu, "The Effect of MyoD Family Proteins on Muscular Atrophy Induced by Brachial Plexus Injury in Rats," Zhonghua Medicine Magazine, Vol. 82, No. 8, 2002, pp. 561-563.

[15] R. N. Cooper, S. Tajbakhsh, V. Mouly, G. Cossu, M. Buckingham and G. S. Butler-Browne, "In Vivo Satellite Cell Activation via Myf5 and MyoD in Regenerating Mouse Skeletal Muscle," Journal of Cell Science, Vol. 112, Pt. 17, 1999, pp. 2895-2901.

[16] M. Lindstrom, F. Pedrosa-Domellof and L. E. Thornell, 
"Satellite Cell Heterogeneity with Respect to Expression of MyoD, Myogenin, Dlk1 and C-Met in Human Skeletal Muscle: Application to a Cohort of Power Lifters and Sedentary Men," Histochemistry and Cell Biology, Vol. 134, No. 4, 2010, pp. 371-385.

[17] M. H. Mokalled, A. N. Johnson, E. E. Creemers and E. N. Olson, "MASTR Directs MyoD-Dependent Satellite Cell Differentiation during Skeletal Muscle Regeneration," Genes \& Development, Vol. 26, No. 2, 2012, pp. 190-202. doi:10.1101/gad.179663.111

[18] P. Seale, J. Ishibashi, C. Holterman and M. A. Rudnicki, "Muscle Satellite Cell-Specific Genes Identified by Genetic Profiling of MyoD-Deficient Myogenic Cell," De- velopmental Biology, Vol. 275, No. 2, 2004, pp. 287-300. doi:10.1016/j.ydbio.2004.07.034

[19] S. Creuzet, L. Lescaudron, Z. Li and J. Fontaine-Perus, "MyoD, Myogenin, and Desmin-Nls-lacZ Transgene Emphasize the Distinct Patterns of Satellite Cell Activation in Growth and Regeneration," Experimental Cell Research, Vol. 243, No. 2, 1998, pp. 241-253. doi:10.1006/excr.1998.4100

[20] R. Macharia, A. Otto, P. Valasek and K. Patel, "Neuromuscular Junction Morphology, Fiber-Type Proportions, and Satellite-Cell Proliferation Rates are Altered in MyoD(-/-) Mice," Muscle \& Nerve, Vol. 42, No. 1, 2010, pp. 38-52. doi:10.1002/mus. 21637 